\title{
High survival rate of $43 \%$ in out-of-hospital cardiac arrest patients in an optimised chain of survival
}

\author{
L. W. Boyce • T. P. M. Vliet Vlieland • J. Bosch $\cdot$ R. \\ Wolterbeek • G. Volker • H. J. van Exel • C. Heringhaus • \\ M. J. Schalij • P. H. Goossens
}

Published online: 18 October 2014

(C) The Author(s) 2014. This article is published with open access at Springerlink.com

\begin{abstract}
Aims Survival to hospital discharge after out-of-hospital cardiac arrest (OHCA) varies widely. This study describes shortterm survival after OHCA in a region with an extensive care path and a follow-up of 1 year.

Methods Consecutive patients $\geq 16$ years admitted to the emergency department between April 2011 and December 2012 were included. In July 2014 a follow-up took place.
\end{abstract}

L. W. Boyce · T. P. M. Vliet Vlieland · G. Volker · H. J. van Exel •

P. H. Goossens

Rijnlands Rehabilitation Centre, Wassenaarseweg 501,

2333AL Leiden, the Netherlands

T. P. M. Vliet Vlieland

Department of Orthopaedics, Leiden University Medical Center,

Albinusdreef 2, 2333 ZA Leiden, the Netherlands

J. Bosch

Emergency Medical Service Hollands Midden,

Vondellaan 43, 2332 AA Leiden, the Netherlands

R. Wolterbeek

Department of Medical Statistics, Leiden University Medical Center, Einthovenweg 20, 2333 ZC Leiden, the Netherlands

H. J. van Exel • M. J. Schalij

Department of Cardiology, Leiden University Medical Center,

Albinusdreef 2, 2333 ZA Leiden, the Netherlands

C. Heringhaus

Department of Accident and Emergency, Leiden University Medical

Center, Albinusdreef 2, 2333 ZA Leiden, the Netherlands

\section{P. H. Goossens}

Department of Rehabilitation, Leiden University Medical Center,

Albinusdreef 2, 2333 ZA Leiden, the Netherlands

L. W. Boyce $(\bowtie)$

Rijnlands Rehabilitation Centre, PO Box 176, 2300 AD Leiden, the Netherlands

e-mail: 1ww@rrc.nl
Socio-demographic data, characteristics of the OHCA and interventions were described and associations with survival were determined.

Results Two hundred forty-two patients were included (73\% male, median age 65 years). In $76 \%$ the cardiac arrest was of cardiac origin and $52 \%$ had a shockable rhythm. In $74 \%$ the cardiac arrest was witnessed, $76 \%$ received bystander cardiopulmonary resuscitation and in $39 \%$ an automatic external defibrillator (AED) was used. Of the 168 hospitalised patients, 144 underwent therapeutic procedures. A total of 105 patients survived until hospital discharge. Younger age, cardiac arrest in public area, witnessed cardiac arrest, cardiac origin with a shockable rhythm, the use of an AED, shorter time until return of spontaneous circulation, Glasgow Coma Scale (GCS) $\geq 13$ during transport and longer length of hospital stay were associated with survival. Of the 105 survivors 72 survived for at least 1 year after cardiac arrest and 6 patients died.

Conclusion A survival rate of $43 \%$ after OHCA is achievable. Witnessed cardiac arrest, cardiac cause of arrest, initial cardiac rhythm and GCS $\geq 13$ were associated with higher survival.

Keywords Out of hospital cardiac arrest · Survival · Cardiopulmonary resuscitation

\section{Introduction}

Out-of-hospital cardiac arrest (OHCA) is one of the main causes of death in Europe. A systematic review including 67 peer-reviewed studies published from 1990 to 2008 concludes that the incidence of emergency medical service (EMS) attended OHCA in Europe is 86.4 per 100,000 inhabitants per year [1]. That review reports that $60 \%$ of the patients in Europe are treated by EMS after OHCA and $9 \%$ of these patients survive to hospital discharge. In the Netherlands survival rates seem to be relatively high: a study on EMS- 
attended OHCA between 2005 and 2008 reported a survival rate until discharge of $14 \%$ [2]. In 1998, a survival rate of $36 \%$ for EMS-attended OHCA was found in the Amsterdam region [3]. However, that study only included patients who had had an OHCA due to a primary cardiac cause.

Factors positively associated with short-term survival after OHCA described in two systematic reviews are: younger age, male gender, witnessed OHCA, early start of cardiopulmonary resuscitation (CPR), initial rhythm of ventricular fibrillation (VF), the use of an automatic external defibrillator (AED), short time until arrival of ambulance, no EMS intubation and short time until return of spontaneous circulation (ROSC) [4, 5]. Inhospital factors important for survival include therapeutic hypothermia and the availability for acute cardiac interventions 24/7 [6,7]. Revascularisation procedures and the use of an implantable cardioverter defibrillator (ICD) mainly reduce long-term mortality [8].

In the Leiden region efforts are taken to provide an optimal chain for OHCA patients, including optimisation of acute care, treatment during transport, treatment in hospital and cardiac rehabilitation. This study describes survival in an optimised chain for OHCA patients and a follow-up of at least 1 year.

\section{Methods}

In the Leiden area post-cardiac arrest care is organised around one regional cardiac centre (The Leiden University Medical Center, LUMC), where cardiac procedures can be performed $24 / 7$. This centre has an affiliated area of $540 \mathrm{~km}^{2}$ with 542,000 inhabitants. [Statistics Netherlands 2012, www.cbs. nl]. The 112 emergency service alerts the regional ambulance service and other first responders, all equipped with an AED and trained personnel.

Chest compressions performed by the ambulance service are standardised using the Lund University Cardiac Arrest System (LUCASTM, Jolife AB/Physio-Control Lund, Sweden). The ambulance service transports all patients with a chance of survival to the emergency department (ED) of the LUMC (approximately $70 \%$ of the cases; personal communication). If ROSC is achieved, patients are transported to the coronary care unit (CCU) or intensive care unit (ICU). In accordance with guidelines, eligible patients receive mild hypothermia.

This retrospective study included patients $\geq 16$ years, resuscitated outside the hospital and admitted to the ED of the LUMC (April 2011-January 2013). In July 2014 a follow-up took place, including only patients who were discharged alive and who had follow-up treatment in the LUMC. Eligible patients were identified using the electronic diagnosis registry of the LUMC. To ensure no patients were missed, patient selection was checked with the registries of the ambulance service. Patients were excluded if the ambulance service decided not to transport the patient to the ED, if the collapse was not caused by cardiac arrest or when insufficient information $(<50 \%)$ was available from the medical records.

Data were retrieved using a standardised form. A second researcher checked the data of $10 \%$ randomly selected patients. No major differences were found.

The following data were extracted from the medical records: socio-demographic data (gender and age at time of the cardiac arrest); characteristics of the cardiac arrest: cardiac or non-cardiac (trauma, drowning, intoxication, asphyxia, hypovolaemia or other), home or public area (street, work, sports, ambulance), witnessed or not, CPR bystander or first responder and use of AED; treatment and course (the number of shocks provided by EMS), initial cardiac rhythm (shockable or non-shockable) and the interval between collapse and ROSC; the Glasgow Coma Scale (GCS) during ambulance transport or if not available at arrival to the ED; (sub)acute treatment in the hospital, number of days in hospital, hospital survival and discharge destination. Data for the follow-up were extracted from the medical records (LUMC): Survival (dead or alive); if alive: time since cardiac arrest (months); if deceased: time from cardiac arrest until death (days).

All statistical analyses were performed using the SPSS 19 software package. Descriptive statistics were used for the characteristics of the participants. Characteristics of survivors and non-survivors were compared by unpaired t-tests (MannWhitney U test) or Chi square tests, where appropriate. Factors associated with survival $(p<0.05)$ were entered into bivariate and multivariate logistic regression analyses, with survival until hospital discharge as dependent variable. By backward elimination, variables that lacked independent association were removed.

This retrospective study (chart review only) falls outside the remit of the Dutch Medical Research Involving Human Beings Act (Medical Ethical Review Board of the LUMC).

\section{Results}

Patients

In the study period 263 patients were identified. After examination of the medical records 21 patients were excluded (Fig. 1).

Table 1 shows the socio-demographic characteristics, characteristics of the medical condition and treatment and course of the 242 included patients. Their median age was 65 years (range 20-95) and 73\% were male. Of the OHCAs 67\% were witnessed by bystanders and $9 \%$ by EMS personnel. A total 
Fig. 1 Flow chart of patients after $\mathrm{OHCA}$

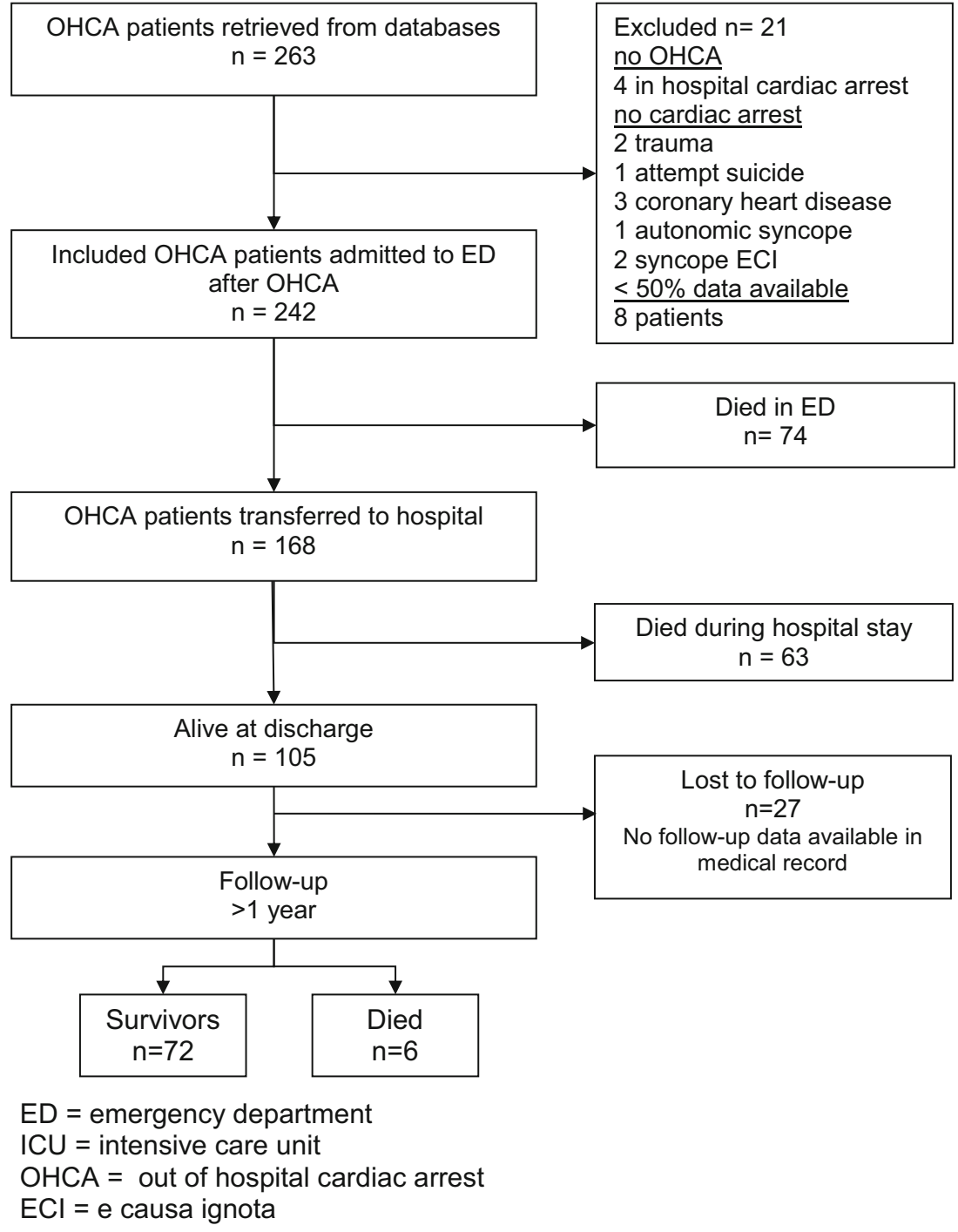

of $76 \%$ of the patients received bystander CPR and in nearly $40 \%$ an AED was used. Most of the arrests took place at home. The majority of the cardiac arrests were of cardiac origin with a shockable rhythm in about half of all patients at EMS arrival.

\section{Survival and discharge destination}

Of the 242 patients who attended the ED, 74 (31\%) died on the emergency ward. Four of the ED stabilised patients were immediately transferred to another hospital. Of the remaining 164 patients, 63 patients died in hospital, on average 2 days after OHCA. After stabilisation on the CCU/ICU 36 patients were transferred to another hospital. In total 105 patients (43\%) survived to hospital discharge. Their hospital stay was on average 9 days. Three were discharged to a nursing facility and 66 patients went home.
Factors associated with survival

Table 1 shows the characteristics of the 105 patients who survived until hospital discharge and the 137 patients who did not. Survivors were significantly younger, significantly more often had a cardiac arrest in a public area, a witnessed arrest, an arrest of cardiac origin and a shockable rhythm, AED was more often used, a shorter time until ROSC, a GCS $\geq 13$ during ambulance transport post CPR and a longer length of hospital stay. No significant associations with survival were found for gender, bystander CPR, number of shocks and witnessed monitored cardiac arrest.

In the multivariate analyses, the variables location and use of AED were left out because of lack of independent association in the bivariate models. The time until ROSC was removed because of its almost linear relationship with the dependent variable survival. Logistic regression shows that four of the variables contribute significantly to surviving 
Table 1 Characteristics of 242 patients who survived out-ofhospital cardiac arrest
${ }^{\text {a }}$ P-value with Chi-square or Ttest

* Patients could undergo more than one (sub)acute intervention

$C A$ Cardiac arrest, CPR Cardiopulmonary resuscitation, $A E D$ Automatic external defibrillator, ROSC Return of spontaneous circulation, $P C I$ Percutaneous coronary intervention, $C A B G$ Coronary artery bypass graft, ICD Implantable cardioverter defibrillator

\begin{tabular}{|c|c|c|c|c|c|c|c|}
\hline & \multicolumn{2}{|c|}{ All patients $n=242$} & \multicolumn{2}{|c|}{$\begin{array}{l}\text { Survivors } \\
n=105(43 \%)\end{array}$} & \multicolumn{2}{|c|}{$\begin{array}{l}\text { Non-survivors } \\
n=137(57 \%)\end{array}$} & \multirow[t]{2}{*}{$P$ value $^{\mathrm{a}}$} \\
\hline & $\mathrm{n}$ & & $\mathrm{n}$ & & $\mathrm{n}$ & & \\
\hline Age in years, mean (SD) & & $64.8(14.7)$ & & $61.5(13.7)$ & & $67.4(15)$ & .002 \\
\hline Male gender, n (\%) & & $176(73)$ & & $77(73)$ & & $99(72)$ & .853 \\
\hline \multicolumn{8}{|l|}{ Location of CA, n (\%) } \\
\hline In/around home & 241 & $148(61)$ & 105 & $47(45)$ & 136 & $101(74)$ & $<.001$ \\
\hline Public area & & $93(39)$ & & $58(55)$ & & $35(26)$ & $<.001$ \\
\hline \multicolumn{8}{|l|}{ Cause CA, n (\%) } \\
\hline Cardiac & 240 & $183(76)$ & 104 & $93(89)$ & 136 & $90(66)$ & $<.001$ \\
\hline Non-cardiac & & $57(24)$ & & $11(11)$ & & $46(34)$ & $<.001$ \\
\hline \multicolumn{8}{|l|}{ Witnessed CA, n (\%) } \\
\hline Yes & 237 & $159(67)$ & 103 & $82(80)$ & 134 & $77(57)$ & $<.001$ \\
\hline \multicolumn{8}{|l|}{ CPR, n (\%) } \\
\hline CPR bystander & 240 & $123(51)$ & 104 & $57(55)$ & 136 & $66(49)$ & .335 \\
\hline CPR first responder & & $61(25)$ & & $27(26)$ & & $34(25)$ & .865 \\
\hline Emergency medical service & & $56(23)$ & & $20(19)$ & & $36(27)$ & .189 \\
\hline \multicolumn{8}{|l|}{$\mathrm{AED}, \mathrm{n}(\%)$} \\
\hline Yes & 240 & $94(39)$ & 104 & $53(51)$ & 136 & $41(30)$ & .001 \\
\hline \multicolumn{8}{|l|}{ Monitored arrest, $\mathrm{n}(\%)$} \\
\hline Yes & 242 & $21(9)$ & 105 & $11(11)$ & 137 & $10(7)$ & .384 \\
\hline \multicolumn{8}{|l|}{ Initial cardiac rhythm, $\mathrm{n}(\%)$} \\
\hline Shockable & 237 & $145(61)$ & 102 & $88(86)$ & 135 & $57(42)$ & $<.001$ \\
\hline Non-shockable & & $92(39)$ & & $14(14)$ & & $78(58)$ & $<.001$ \\
\hline Number of defibrillations & & $2.5( \pm 3)$ & & $2.3( \pm 2)$ & & $2.7( \pm 3.5)$ & .326 \\
\hline \multicolumn{8}{|l|}{ Interval collapse - ROSC, n (\%) } \\
\hline No ROSC & 221 & $35(16)$ & 95 & $0(0)$ & 126 & $35(28)$ & $<.001$ \\
\hline$<6$ min. & & $74(33)$ & & $62(65)$ & & $12(10)$ & $<.001$ \\
\hline $6-10 \mathrm{~min}$. & & $18(8)$ & & $10(11)$ & & $8(6)$ & .261 \\
\hline$>10 \mathrm{~min}$ & & $94(43)$ & & $23(24)$ & & $71(56)$ & $<.001$ \\
\hline \multicolumn{8}{|l|}{ Glasgow Coma Scale, n (\%) } \\
\hline Minor $\geq 13$ & 196 & $14(7)$ & 69 & $13(19)$ & 127 & $1(1)$ & $<.001$ \\
\hline Moderate 9-12 & & $6(3)$ & & $5(7)$ & & $1(1)$ & .012 \\
\hline Severe $<9$ & & $176(90)$ & & $51(74)$ & & $125(98)$ & $<.001$ \\
\hline \multicolumn{8}{|l|}{ Cardiac intervention $*, \mathrm{n}(\%)$} \\
\hline PCI & 242 & $73(30)$ & 105 & $55(52)$ & 137 & $18(13)$ & \\
\hline CABG & & $12(5)$ & & $11(10)$ & & $1(1)$ & \\
\hline $\mathrm{ICD}$ & & $26(11)$ & & $26(25)$ & & $0(0)$ & \\
\hline Therapeutic hypothermia & & $94(39)$ & & $49(47)$ & & $45(33)$ & \\
\hline Length hospital stay (days) & & $5( \pm 8.2)$ & & $9( \pm 8.7)$ & & $2( \pm 6.3)$ & $<.001$ \\
\hline
\end{tabular}

OHCA: witnessed cardiac arrest, cardiac cause, initial rhythm and GCS $\geq 13$ (Table 2).

\section{Follow-up}

For 78 of the 105 patients who survived until hospital discharge, follow-up data of at least 1 year after cardiac arrest were available. Of these patients $72(92 \%)$ were alive at least
1 year after cardiac arrest. Six patients died whereas the medical records of 27 patients were not available. The main reason patients were lost to follow-up was that they were living outside the Leiden region. Of the 72 survivors the average survival time was 28.6 months (SD 6.3) after the cardiac arrest 23 patients survived to $0-24$ months, 38 patients survived to 24 and 36 months and 11 patients were still alive $>36$ months after cardiac arrest. All 6 patients who died after 
Table 2 Logistic regression on the likelihood of survival in OHCA patients

\begin{tabular}{llllr}
\hline & p-value & Odds ratio & \multicolumn{2}{c}{$95 \%$ CI for odds ratio } \\
\cline { 3 - 5 } & & & Lower & Upper \\
\hline Age & & & 0.950 & 1.002 \\
Witnessed CA & 0.019 & 2.851 & 1.187 & 6.849 \\
Cardiac cause of arrest & 0.009 & 5.947 & 1.569 & 22.549 \\
ICR shockable & 0.017 & 2.887 & 1.205 & 6.917 \\
GCS & 0.000 & 1.498 & 1.202 & 1.867 \\
\hline
\end{tabular}

$C A$ Cardiac arrest, C.I. Confidence interval, GCS Glasgow coma scale, ICR Initial cardiac rhythm

hospital discharge did so within a month after their cardiac arrest (5-25 days after cardiac arrest).

\section{Discussion}

This study shows that a survival rate until hospital discharge of $43 \%$ of EMS-treated OHCA patients is feasible in an optimised chain of survival. The survival rate is higher than the average $14 \%$ as reported in Europe $[1,2]$. However, a study in the Netherlands by Waalewijn et al.[3] also reported a high survival rate (36\%) of EMS-attended OHCA, be it that that study only included OHCA of cardiac origin whereas this study included all EMS-treated patients. In approximately $70 \%$ of the cases the EMS decided to transport patients to the hospital (unpublished data), which is comparable with the $60 \%$ EMS treatment found in the literature [1].

In this study survivors were younger, more often had a witnessed cardiac arrest, a cardiac origin of the arrest and a shockable rhythm compared with those who died. As expected, non-comatose patients (GCS $\geq 13$ post $\mathrm{CPR}$ ) and patients with sustained ROSC in the ambulance or ED had better chances of survival [4].

To find an explanation for the high survival rate, a comparison with the patient characteristics and treatment in the literature was made. The mean age (64.8 year) of the patients in the present study was in the same range as those seen in previous studies (64-67 years) $[3,5,6]$. In our study a relatively high proportion $(67 \%)$ of the OHCAs were witnessed and this was positively correlated with survival. We postulate that witnessing an OHCA contributes more to survival than CPR itself, since witnessed arrests give a higher chance of early alarm and use of an AED within minutes after the collapse.

A cardiac cause of cardiac arrest with an initial shockable rhythm may also partly explain the favourable outcome of this study. A systematic review reported that patients with VF or VT had a survival chance of 1 in every 4 to 7 patients compared with only 1 in every 21 to 500 patients in whom the first rhythm was asystole [4]. In the current study, a high percentage (49\%) of patients showed an initial cardiac rhythm of VF, whereas other studies reported that only $30 \%$ of the patients had an initial rhythm of VF [9]. Since VF is only recorded in the acute stage after cardiac arrest, a high percentage of VF might indicate short arrival times of the ambulance service.

The use of an on-site AED doubles survival, probably caused by the reduction in time to first shock [9]. Berdowski found that an AED was used in $21 \%$ of the cases [10]. In the current study AED was used in $39 \%$ of the cases. It is plausible that the availability of AEDs has grown in the last 5 to 10 years, contributing to higher survival rates.

Another factor that might positively influence survival is the regional function of the LUMC for all cardiac arrests. Studies have described that lower mortality is seen in hospitals that treat a high volume of cardiac arrests and who provide $24 \mathrm{~h}$ interventional cardiac services $[5,6]$.

In this study $86 \%$ of the patients who reached the $\mathrm{CCU}$ or ICU received on average 1.4 (sub)acute interventions per person. A comparison with the literature was not possible, since no studies on incidence of interventions in a comparable group were found. It is plausible that only patients with fair chances of survival receive interventions.

Of the 73 patients who underwent PCI 55 survived until hospital discharge and 18 died in hospital. Of the 12 coronary artery bypass graft procedures one patient died in hospital. Of the 26 patients who received an ICD during initial hospitalisation and the 34 patients who received an ICD after hospital discharge two patients died within a year after the cardiac arrest. The positive influence of an ICD in reducing mortality in the long term is described in a clinical review by Arrawwawala et al. [8]. Of the 95 patients who did not receive any intervention 80 died before hospital discharge. Follow-up data of $74 \%$ of the patients were available, suggesting that after an average follow-up of 28.6 months the large majority were alive. However, as the medical records from many patients were not available, results have to be interpreted with caution.

All EMS-treated survivors of OHCA with a cardiac cause need cardiac rehabilitation. A recent Dutch study concluded that only $29 \%$ of the patients eligible for cardiac rehabilitation received cardiac rehabilitation [11]. With increasing survival rates probably more effort should be put into aligning the process of cardiac rehabilitation for OHCA survivors.

\section{Conclusions}

This study showed a survival rate of $43 \%$ after OHCA in a urban region in the Netherlands where an optimised chain of acute and sub-acute treatment exists. 
Witnessed cardiac arrest, cardiac origin of the arrest, shockable initial rhythm and GCS $>13$ post CPR were independently related to survival to hospital discharge. Availability of AED, short arrival times of EMS and (sub)acute treatment may also contribute to the success rate, but more research into the extent of the effect on survival is needed.

Funding None.

\section{Conflicts of interest None declared}

Open Access This article is distributed under the terms of the Creative Commons Attribution License which permits any use, distribution, and reproduction in any medium, provided the original author(s) and the source are credited.

\section{References}

1. Berdowski J, Berg R, Tijssen JGP, et al. Global incidences of out-ofhospital cardiac arrest and survival rates: systematic review of 67 prospective studies. Resuscitation. 2010;81(11):1479-87. doi:10. 1016/j.resuscitation.2010.08.006.

2. Hulleman M, Berdowski J, de Groot, et al. Implantable cardioverterdefibrillators have reduced the incidence of resuscitation for out-ofhospital cardiac arrest caused by lethal arrhythmias. Circulation. 2012;126(7):815-21. doi:10.1161/CIRCULATIONAHA.111. 089425.

3. Waalewijn R, de Vos R, Koster RW. Out-of-hospital cardiac arrests in Amsterdam and its surrounding areas: results from the Amsterdam resuscitation study (ARREST) in "Utstein" style. Resuscitation. 1998;38(3):157-67.

4. Sasson C, Rogers MAM, Dahl J, et al. Predictors of survival from out-of-hospital cardiac arrest: a systematic review and meta-analysis. Circ Cardiovasc Qual Outcomes. 2010;3(1):63-81. doi:10.1161/ CIRCOUTCOMES.109.889576.

5. Stub D, Smith K, Bray JE, et al. Hospital characteristics are associated with patient outcomes following out-of-hospital cardiac arrest. Heart. 2011;97(18):1489-94. doi:10.1136/hrt. 2011.226431.

6. Johnson NJ, Salhi RA, Abella BS, et al. Emergency department factors associated with survival after sudden cardiac arrest. Resuscitation. 2012;84(3):292-7. doi:10.1016/j.resuscitation.2012. 10.013 .

7. Six AJ, Backus BE, Kingma A, et al. Consumption of diagnostic procedures and other cardiology care in chest pain patients after presentation at the emergency department. Neth Heart J. 2012;20(12):499-504. doi:10.1007/s12471-012-03226.

8. Arawwawala D, Brett SJ. Clinical review: beyond immediate survival from resuscitation-long-term outcome considerations after cardiac arrest. Crit Care. 2007;11(6):235. doi:10.1186/cc6139.

9. Weisfeldt ML, Sitlani CM, Ornato, et al. Survival after application of automatic external defibrillators before arrival of the emergency medical system: evaluation in the resuscitation outcomes consortium population of 21 million. J Am Coll Cardiol. 2010;55(16):1713-20.

10. Berdowski J, Blom MT, Bardai A, et al. Impact of onsite or dispatched automated external defibrillator use on survival after out-of-hospital cardiac arrest. Circulation. 2011;124(20):2225-32. doi:10.1161/CIRCULATIONAHA.110.015545.

11. Van Engen-Verheul M, de Vries H, Kemps H, et al. Cardiac rehabilitation uptake and its determinants in the Netherlands. Eur J Prev Cardiol. 2013;20(2):349-56. doi:10.1177/ 2047487312439497. 